\title{
Partitioning of on-demand electron pairs
}

\author{
Niels Ubbelohde ${ }^{1 \star}$, Frank Hohls², Vyacheslavs Kashcheyevs ${ }^{3}$, Timo Wagner', Lukas Fricke ${ }^{2}$, \\ Bernd Kästner ${ }^{2}$, Klaus Pierz ${ }^{2}$, Hans W. Schumacher ${ }^{2}$ and Rolf J. Haug ${ }^{1}$
}

The on-demand generation and separation of entangled photon pairs are key components of quantum information processing in quantum optics ${ }^{1-3}$. In an electronic analogue, the decomposition of electron pairs represents an essential building block for using the quantum state of ballistic electrons in electron quantum optics ${ }^{4-7}$. The scattering of electrons has been used to probe the particle statistics of stochastic sources in Hanbury Brown and Twiss experiments ${ }^{8,9}$ and the recent advent of on-demand sources further offers the possibility to achieve indistinguishability between multiple sources in Hong-Ou-Mandel experiments ${ }^{10-15}$. Cooper pairs impinging stochastically at a mesoscopic beamsplitter have been successfully partitioned, as verified by measuring the coincidence of arrival ${ }^{16-21}$. Here, we demonstrate the splitting of electron pairs generated on demand. Coincidence correlation measurements allow the reconstruction of the full counting statistics, revealing regimes of statistically independent, distinguishable or correlated partitioning, and have been envisioned as a source of information on the quantum state of the electron pair 22-26. The high pair-splitting fidelity opens a path to future on-demand generation of spin-entangled electron pairs from a suitably prepared two-electron quantum-dot ground state.

The few-electron source is based on a single-parameter non-adiabatic quantized charge pump ${ }^{27-29}$, which enables the deterministic generation of single electrons and electron pairs with tunable emission energy ${ }^{12,30}$. Non-equilibrium electrons propagate along the edge of a quantum Hall sample with minimal inelastic scattering. The device and measurement set-up are presented in Fig. 1. An energy-selective detector barrier splits the incoming beam of electrons into two detector paths. The coincidence of arrival of electrons in the two detector channels leads to positive correlation between the time-dependent current signals. These correlations are inferred from a measurement of the zero-frequency cross-correlation shot noise. Although an oscillator-controlled electron source is noiseless ${ }^{31}$, the splitting of electron pairs generates partitioning noise and enables tomography of the probability distribution for the partitioning outcomes within each emission cycle.

The generation and energy-selective detection of on-demand non-equilibrium electrons was demonstrated with the electron source configured to emit one electron with charge $e$ and repetition frequency $f$ of $280 \mathrm{MHz}$. Figure 2 shows the transmitted current $I_{\mathrm{T}}$ as a function of barrier energy. The maximum level of $I_{\mathrm{T}}$ is $2 \%$ below the emission current $I_{\mathrm{P}}=1$ ef due to residual inelastic scattering events on the $2-\mu \mathrm{m}$-long path to the barrier (the emission error of the source is $\left.<1 \times 10^{-4}\right)$. For energies greater than $57 \mathrm{meV}$, the current is pinched off as all of the emitted electrons are reflected at the beamsplitter. The emission energy is defined by the exit barrier height and can also be modified by the amplitude and shape of the driving waveform, which additionally influence the width of the energy distribution ${ }^{30}$.

Figure 2 also shows the cross-correlation noise power $S_{\mathrm{X}}$. Because the sequence of emitted electrons is very regular and does not a

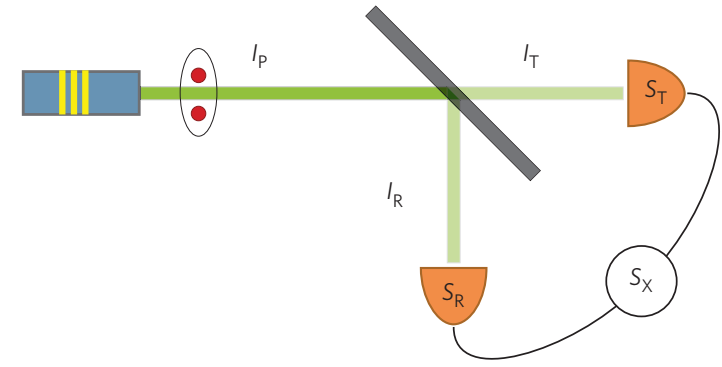

b
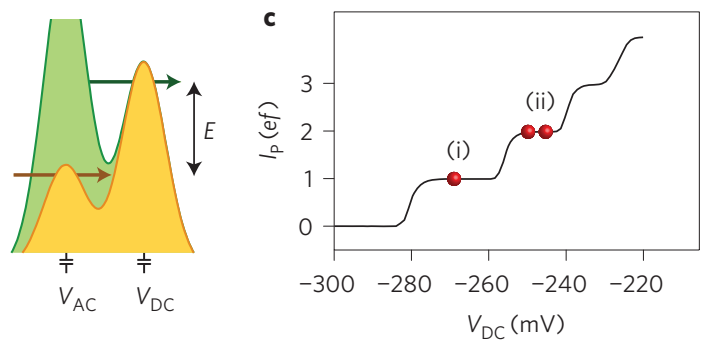

d

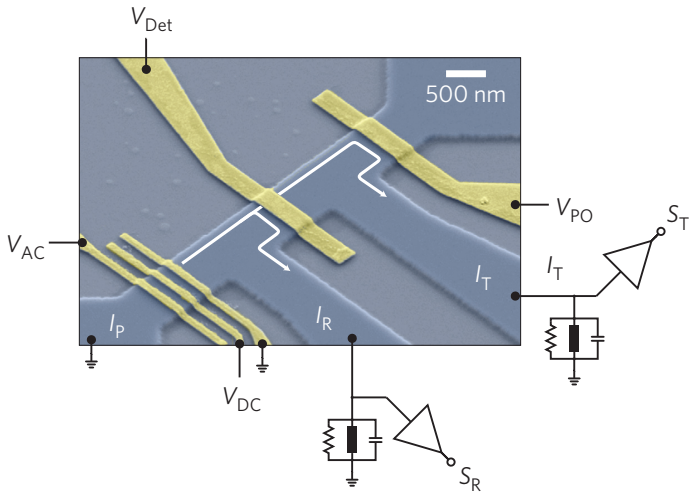

Figure 1 | Measurement set-up. a, Schematic diagram of the experimental set-up following the Hanbury Brown and Twiss geometry. The current $I_{p}$ of the triggered electron source is split into a reflected part $I_{R}$ and a transmitted part $I_{T}$. To measure the coincidence of arrival, the crosscorrelation between the time-dependent fluctuations $I_{R}(t)$ and $I_{T}(t)$ is detected, giving rise to the cross-correlation noise power $S_{X}$ and the singledetector channel noise power $S_{R}$ and $S_{T}$. $\mathbf{b}$, Energy diagram of the loading (orange) and unloading (green) phase of the dynamic quantum dot, resulting in a quantized current $I_{\mathrm{p}}$. c, Typical current characteristic versus exit gate voltage $\left(V_{D C}\right)$, showing plateaux corresponding to the emission of single electrons (i) and electron pairs (ii). d, Micrograph of the sample geometry. The gates controlled with voltages $V_{A C}$ and $V_{D C}$ form the electron source. White lines indicate chiral edge channels, which guide the electrons to the detector barrier defined by $V_{\text {Det. }}$. The channel exit is pinched off $\left(V_{\mathrm{PO}}\right)$, ensuring that all transmitted electrons are recorded by $I_{\mathrm{T}}$. 


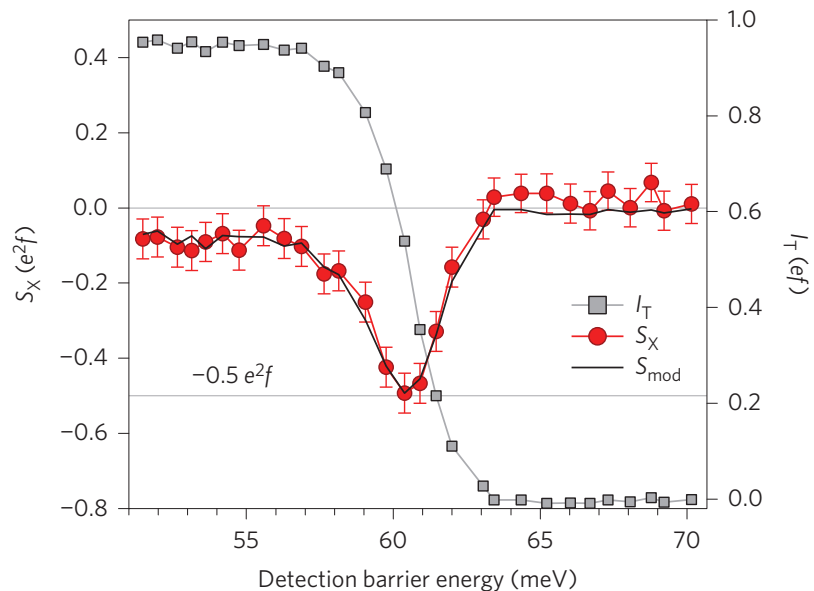

Figure $\mathbf{2}$ | Partitioning noise of an on-demand electron source. Transmitted current $I_{T}$ and cross-correlation noise power $S_{X}$ as a function of the energetic detector barrier height with the electron source configured to emit a sequence of single electrons. $0.5 \mathrm{e}^{2} \mathrm{f}$ corresponds to a noise power of $3.6 \times 10^{-30} \mathrm{~A}^{2} \mathrm{~Hz}^{-1}$. Error bars consist of the statistical error, the error of the estimated background and the calibration error. The solid black line represents the expected value $S_{\text {mod }}$ of the partitioning noise determined by the transmission probability $T(E)$, which in turn is given by the fraction of the transmitted current. fluctuate within the resolution limit of our noise set-up, the correlation between the two detectors is determined solely by the partitioning of the electrons. For energies below the pinch-off region we observe a small background of partitioning noise due to the $2 \%$ reflection of the energy-relaxed electrons at the detection barrier. When all electrons are reflected at large detector barrier energies, the cross-correlation signal is zero. In the transition, $S_{\mathrm{X}}$ reaches a value of $-0.5 e^{2} f$ due to the statistical partitioning at the partially transparent barrier.

For one-electron scattering the current can be equated with the transmission probability $T(E)$ as a function of the electron energy $I_{\mathrm{T}}(E)=T(E) e f$, and the resulting expected value for the partitioning noise, $S_{\text {mod }}=-2 T(1-T) e^{2} f$ (black line, Fig. 2), agrees very well with the measured data. In particular, the negative sign of the cross-correlation signal reflects the anticorrelation of the two detector channels, as the sequence of single electrons is partitioned into either reflected or transmitted electrons.

In the following, we characterize the on-demand emission and splitting of electron pairs. With the quantum-dot current tuned on the 2 ef plateau, one electron pair is emitted per waveform cycle. The corresponding partitioning results are shown in Fig. 3a. The noise power shows a single dip with twice the amplitude of that observed for the single-electron case (Fig. 2). For a clocked stream of electron pairs, the full counting statistics of transmission ${ }^{24}$ can be unambiguously derived from the measurement of $I_{\mathrm{T}}$ and $S_{\mathrm{X}}$, where $I_{\mathrm{T}}=\left(p_{1}+2 p_{2}\right) e f, S_{\mathrm{X}}=-2\left[p_{0}\left(1-p_{0}\right)+p_{2}\left(1-p_{2}\right)+2 p_{0} p_{2}\right] e^{2} f$
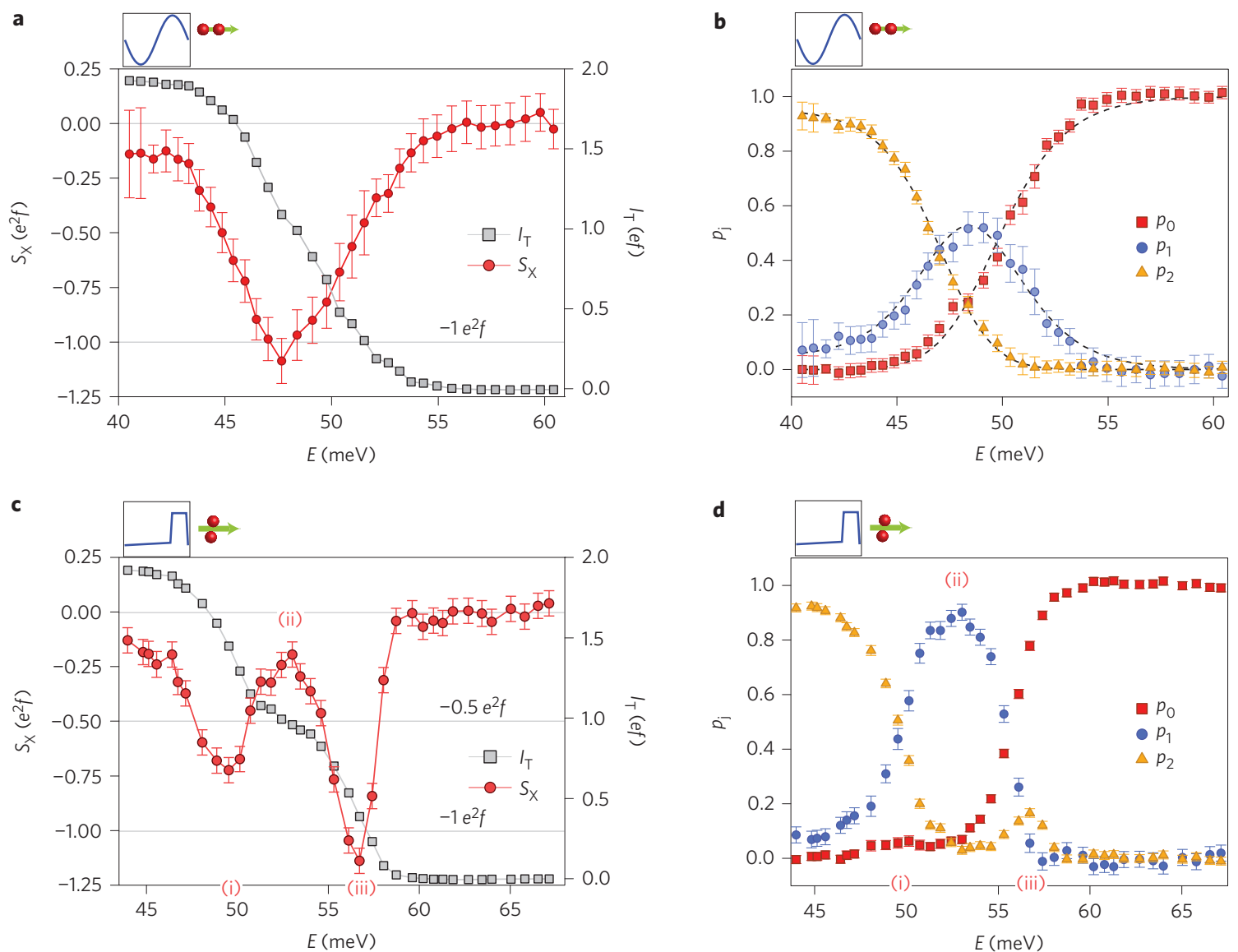

Figure 3 | Partitioning of on-demand electron pairs. a, Transmitted current and cross-correlation noise power for a source of electron pairs emitted slowly by a sinusoidal gate drive (see pictogram). The horizontal line at $e^{2} f$ indicates the level for the superimposed noise amplitude of two partitioned electrons. b. Probabilities for two transmitted (reflected) electrons $p_{2}\left(p_{0}\right)$ and for split electron pairs $p_{1}$. The dashed lines represent fits to the data using a binomial distribution. c,d, Electron source tuned to emit electrons at separate energies due to a gate drive with fast emission pulse (pictogram shows the waveform of $-V_{\mathrm{AC}}$ ) (c) leading to a splitting fidelity of $90 \%$ and an increased bunching at $57 \mathrm{meV}\left(p_{2}\right)$ and $50 \mathrm{meV}\left(p_{0}\right)$ (d). (i), (ii) and (iii) mark peak positions. 


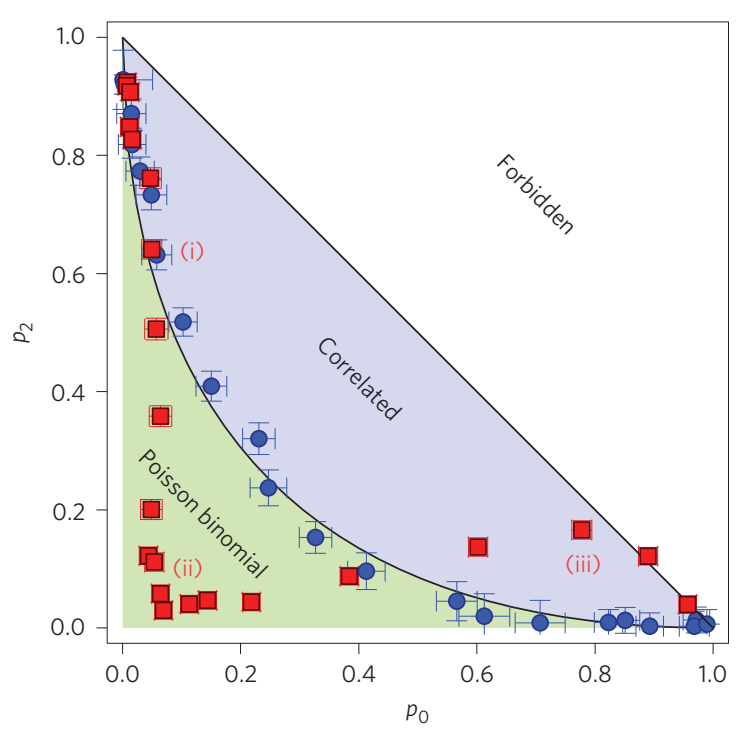

Figure 4 | Regimes of binomial distribution. $p_{2}$ as a function of $p_{0}$ for the two-electron partitioning of Fig. 3b (blue circles) and Fig. 3d (red squares). The line $\sqrt{p_{0}}+\sqrt{p_{2}}=1$ separates the domains of necessarily correlated and potentially independent partitioning. (i), (ii) and (iii) mark data points corresponding to the peaks in Fig. 3c,d.

and $\sum_{i} p_{i}=1 . p_{2}$ and $p_{0}$ denote the probability that both electrons are transmitted or reflected, respectively. $p_{1}$ is the probability for the electron pair to split and both detector channels to record one electron each (Fig. 3b). For sufficiently slow driving of the source, the two electrons are expected to be emitted in sequence at the same energy (Supplementary Section A2). Subsequent independent scattering with the same probability $T$ for each electron results in a binomial partitioning distribution, $p_{2}=T^{2}$ and $p_{0}=(1-T)^{2}$. Assuming a simple step function $T(E)=1 /\left\{1+\exp \left[\left(E-E_{0}\right) / \Delta_{\mathrm{b}}\right]\right\}$, we derive the probabilities shown in Fig. $3 \mathrm{~b}$ by black dashed lines, with energy $E_{0}=48.4 \mathrm{meV}$ and the detected energy broadening $\Delta_{\mathrm{b}}=1.8 \mathrm{meV}$ of the emitted electrons as fit parameters and consistent with the normalization of the probabilities to unity. A monotonic energy dependence of the barrier yields good agreement with the measurement, and at $T=0.5$ the maximum probability for splitting the electron pair is $p_{1}=50 \%$.

A particular advantage of single-parameter charge pumps operated far from equilibrium is the possibility to separately control loading from the source and emission into the drain by appropriate tuning of the driving waveform ${ }^{29}$. We now employ a sharp ejection pulse for the emission part of the cycle, aiming to reduce the time delay between the electrons and to preserve the energy difference imposed by the confinement in the quantum dot in the emission spectrum of ballistically propagating electrons (Supplementary Section A3). The resulting transmitted current shows a plateau at $1 \mathrm{ef}$, hinting at a separation of the two electrons in energy (Fig. 3c). In comparison to the previous measurement, the crosscorrelation noise power now displays two separate dips at transmission current values of 1.5 ef (i) and 0.5 ef (iii). Between these dips, the cross-correlation noise drops to a low absolute value of $0.2 e^{2} f$ at a transmission current of 1 ef (ii), consistent with each of the two detectors receiving a positively correlated, but highly regular stream of one electron per cycle. The corresponding counting statistics (Fig. 3d) show the fidelity of splitting the electron pair $\left(p_{1}\right)$ reaching $90 \%$.

The counting statistics not only allow us to verify the pairsplitting fidelity, but can also provide evidence for electron interactions in the partitioning process. Strong anticorrelation between the paths taken by individual electrons after the pair hits the barrier $\left(p_{1}>50 \%\right)$ indicates that the two electrons are distinguished by the scattering process. Statistically independent scattering of two electrons with transmission probabilities $T_{a}$ and $T_{\mathrm{b}}$ would result in a Poisson binomial distribution, $p_{0}=T_{\mathrm{a}} T_{\mathrm{b}}$, $p_{2}=\left(1-T_{\mathrm{a}}\right)\left(1-T_{\mathrm{b}}\right)$, which obeys the inequality $\sqrt{p_{0}}+\sqrt{p_{2}} \leq 1$. This necessary condition for statistical independence defines a domain in the $\left(p_{0}, p_{2}\right)$ plane, which is compared to the statistics inferred from measurements in Fig. 4. The data points for pair emission, where the electrons are emitted sequentially at the same energy (Fig. 3b, blue circles in Fig. 4), fall on the border of the domain, as expected from $T_{\mathrm{a}}=T_{\mathrm{b}}=T(E)$. The high-fidelity partitioning indicated by the peak in $p_{1}$ in Fig. $3 \mathrm{~d}$ (red squares marked by (ii) in Fig. 4) is consistent with the Poisson binomial distribution for a semitransparent barrier (small $p_{0}, p_{2}$ ), where the electrons are distinguished by $T_{\mathrm{a}} \neq T_{\mathrm{b}}$. Within this region an HBT correlation measurement is unable to discriminate the physical mechanisms of partitioning; a difference in transmission coefficients may indicate splitting in energy or a more complex orbital separation induced by the Pauli principle (for a specific example see Supplementary Section B1). However, for low transmittance (small $p_{2}$, large $p_{0}$ ) the independence condition is violated, indicating an increased bunching of electrons at one detector (co-transmission or reflection) beyond statistical coincidence. This effect is reflected in the cross-correlation shot noise, which significantly exceeds the value of single particle partitioning $\left(0.5 e^{2} f\right)$, suggesting the two electrons are reflected or transmitted together at their respective energies $(E=50 \mathrm{meV}$ and $E=57 \mathrm{meV}$ in Fig. 3c,d, respectively).

The violation of scattering independence requires two-body correlations in the incoming state or in the partitioning process. Considering quantum correlations in the incoming state imposed by the Fermi statistics, we note that a single Slater determinant of two orthogonal spin-orbitals is insufficient for the counting statistics to exceed the binomial constraint (Supplementary Section B2), whereas a spin singlet with additional orbital correlations (for example, a symmetric combination of two orbitals with different energies ${ }^{24,25}$ ) can in principle cause a bunching effect in the transmission. However, the non-monotonic energy dependence of the bunching anomaly (peak (iii) of $p_{2}(E)$ in Fig. $3 \mathrm{~d}$ near $E=57 \mathrm{meV}$ ) is inconsistent with the single-channel Landauer-Büttiker picture and a monotonic $T(E)$, regardless of the initial correlations in the incoming two-electron state, including an arbitrary mixed state (Supplementary Section B3). Hence, we conclude that electron-electron interaction during the partitioning process remains the most probable cause for the observed bunching, which may open a path to introduce nonlinearity in electron quantum optics devices.

\section{Methods}

The electrons were sourced from a dynamic quantum dot formed by two metallic gates over a 900-nm-wide etched channel of a two-dimensional electron gas (2DEG) $90 \mathrm{~nm}$ beneath the surface of a GaAs/AlGaAs heterostructure ${ }^{27-31}$. The carrier mobility and density of the 2DEG were $1.6 \times 10^{6} \mathrm{~cm}^{2} \mathrm{~V}^{-1} \mathrm{~s}^{-1}$ and $2.6 \times 10^{11} \mathrm{~cm}^{-2}$, respectively. Controlled by the modulation of the entrance gate, a defined number of electrons were loaded into the quantum dot and ejected over the exit barrier into the channel (see refs 28-31 for a detailed description of the operating principle). The entrance gate voltage was driven by an arbitrary waveform generator, enabling the triggered emission of electrons. The exit gate was held at a fixed potential. Owing to an applied perpendicular magnetic field of $12 \mathrm{~T}$, the emitted electrons followed the sample edge to a detector barrier, where they were transmitted or reflected into two detector channels depending on their emission energy in relation to the energetic barrier height. The value for the magnetic field was chosen to suppress the energy relaxation of the electrons on the 2 - $\mu \mathrm{m}$-long path towards the detector barrier. For lower values of magnetic field, inelastic scattering set in, dominated by longitudinal optical phonon coupling ${ }^{32,33}$ and visible as steps in the transmitted current with a period of $36 \mathrm{meV}$. The error rates of the one- and two-electron emission were below the measurement resolution and were estimated from the plateau quality to be $<1 \times 10^{-4}$ (ref. 31 ).

Measurement of the transmitted current $I_{\mathrm{T}}$ allowed the average fraction of reflected electrons to be determined. The fluctuations of the transmitted and reflected current were also recorded. The two detectors were formed by parallel 
tank circuits with a resonance frequency of $1 \mathrm{MHz}$, and converted the current fluctuations to voltage fluctuations. To increase the bandwidth of the tank circuits, low-capacitance wires connected the detectors with high-electron-mobility transistors for cryogenic amplification. With the device pinched off, the two detector channels could be calibrated independently, as the thermal noise of the detector circuits as a function of temperature allowed in situ calibration of the amplification chain. The real part of the cross-correlation of the two detector signals was integrated over $20 \mathrm{~min}$ and averaged within a frequency window between $500 \mathrm{kHz}$ and $3 \mathrm{MHz}$, yielding the zero-frequency shot noise power $S_{\mathrm{X}}$, which was determined by the partitioning noise.

The energetic height of the detection barrier was calibrated by driving a constant current across the exit barrier. With the entrance gate held at ground potential, the energy of the electrons emitted into the channel was then controlled by the exit gate and approximately given by the bias voltage $V_{\text {Bias }}$ across the exit gate. The combined measurement of $I_{\mathrm{T}}$ and $V_{\text {Bias }}$ as a function of the voltages applied to the exit gate and the detector gate related the transmission energy of the electrons to the detection gate voltage $V_{\text {Det }}$ (ref. 32). The values for $V_{\text {Bias }}$ and $V_{\text {Det }}$ along a threshold current then yielded the energy calibration of the detection barrier.

The sine-waveform parameters were $V_{\mathrm{AC}}=275 \mathrm{mV}$ PP, with a constant offset of $-368 \mathrm{mV}, V_{\mathrm{DC}}=-247 \mathrm{mV}$ for 1 ef and $V_{\mathrm{DC}}=-232 \mathrm{mV}$ for $2 \mathrm{ef}$. The pulse-waveform $(-V)$ is shown in the pictogram in Fig. $3 \mathrm{c}$ and the voltages are $V_{\mathrm{AC}}=138 \mathrm{mV}_{\mathrm{PP}}$, with a constant offset of $-280 \mathrm{mV}, V_{\mathrm{DC}}=-235 \mathrm{mV}$. We verified that the pulse waveform did not add any additional structure to the energy distribution when emitting only one electron per cycle (Supplementary Section C).

The achievable rise time in our set-up for the time-dependent gating could be estimated by time-domain reflectometry to be $\lesssim 0.1 \mathrm{~ns}$. The estimated transit time between the electron source and the partitioning gate was on the order of $0.01 \mathrm{~ns}$.

Received 28 April 2014; accepted 21 October 2014; published online 1 December 2014

\section{References}

1. Kim, J., Benson, O., Kan, H. \& Yamamoto, Y. A single-photon turnstile device. Nature 397, 500-503 (1999).

2. Stevenson, R. M. et al. A semiconductor source of triggered entangled photon pairs. Nature 439, 179-182 (2006)

3. Lang, C. et al. Correlations, indistinguishability and entanglement in Hong-OuMandel experiments at microwave frequencies. Nature Phys. 9, 345-348 (2013).

4. Yamamoto, M. et al. Electrical control of a solid-state flying qubit. Nature Nanotech. 7, 247-251 (2012).

5. Ji, Y. et al. An electronic Mach-Zehnder interferometer. Nature 422, 415-418 (2003).

6. Neder, I. et al. Interference between two indistinguishable electrons from independent sources. Nature 448, 333-337 (2007).

7. Altimiras, C. et al. Non-equilibrium edge-channel spectroscopy in the integer quantum Hall regime. Nature Phys. 6, 34-39 (2009).

8. Henny, M. et al. The fermionic Hanbury Brown and Twiss experiment. Science 284, 296-298 (1999).

9. Oliver, W. D., Kim, J., Liu, R. C. \& Yamamoto, Y. Hanbury Brown and Twiss-type experiment with electrons. Science 284, 299-301 (1999).

10. Fève, G. et al. An on-demand coherent single-electron source. Science 316, 1169-1172 (2007)

11. Bocquillon, E. et al. Electron quantum optics: partitioning electrons one by one. Phys. Rev. Lett. 108, 196803 (2012).

12. Fletcher, J. D. et al. Clock-controlled emission of single-electron wave packets in a solid-state circuit. Phys. Rev. Lett. 111, 216807 (2013).

13. Liu, R. C., Odom, B., Yamamoto, Y. \& Tarucha, S. Quantum interference in electron collision. Nature 391, 263-265 (1998).

14. Bocquillon, E. et al. Coherence and indistinguishability of single electrons emitted by independent sources. Science 339, 1054-1057 (2013).

15. Dubois, J. et al. Minimal-excitation states for electron quantum optics using levitons. Nature 502, 659-663 (2013).

16. Wei, J. \& Chandrasekhar, V. Positive noise cross-correlation in hybrid superconducting and normal-metal three-terminal devices. Nature Phys 6, 494-498 (2010).
17. Herrmann, L. G. et al. Carbon nanotubes as Cooper-pair beam splitters. Phys. Rev. Lett. 104, 026801 (2010).

18. Hofstetter, L., Csonka, S., Nygard, J. \& Schonenberger, C. Cooper pair splitter realized in a two-quantum-dot Y-junction. Nature 461, 960-963 (2009).

19. Hofstetter, L. et al. Finite-bias Cooper pair splitting. Phys. Rev. Lett. 107, 136801 (2011)

20. Das, A. et al. High-efficiency Cooper pair splitting demonstrated by twoparticle conductance resonance and positive noise cross-correlation. Nature Commun. 3, 1165 (2012).

21. Schindele, J., Baumgartner, A. \& Schönenberger, C. Near-unity Cooper pair splitting efficiency. Phys. Rev. Lett. 109, 157002 (2012).

22. Burkard, G., Loss, D. \& Sukhorukov, E. V. Noise of entangled electrons: bunching and antibunching. Phys. Rev. B 61, R16303 (2000).

23. Samuelsson, P. \& Büttiker, M. Quantum state tomography with quantum shot noise. Phys. Rev. B 73, 041305 (2006).

24. Hassler, F., Lesovik, G. B. \& Blatter, G. Effects of exchange symmetry on full counting statistics. Phys. Rev. Lett. 99, 076804 (2007).

25. Hassler, F. et al. Wave-packet formalism of full counting statistics. Phys. Rev. B 78, 165330 (2008)

26. Wahl, C., Rech, J., Jonckheere, T. \& Martin, T. Interactions and charge fractionalization in an electronic Hong-Ou-Mandel interferometer. Phys. Rev. Lett. 112, 046802 (2014)

27. Blumenthal, M. D. et al. Gigahertz quantized charge pumping. Nature Phys. 3, 343-347 (2007).

28. Kaestner, B. et al. Single-parameter nonadiabatic quantized charge pumping Phys. Rev. B 77, 153301 (2008).

29. Giblin, S. P. et al. Towards a quantum representation of the ampere using single electron pumps. Nature Commun. 3, 930 (2012).

30. Leicht, C. et al. Generation of energy selective excitations in quantum Hall edge states. Semicond. Sci. Technol. 26, 055010 (2011).

31. Maire, N. et al. Noise measurement of a quantized charge pump. Appl. Phys. Lett. 92, 082112 (2008).

32. Taubert, D. et al. Relaxation of hot electrons in a degenerate two-dimensional electron system: transition to one-dimensional scattering. Phys. Rev. B 83, 235404 (2011).

33. Sivan, U., Heiblum, M. \& Umbach, C. P. Hot ballistic transport and phonon emission in a two-dimensional electron gas. Phys. Rev. Lett. 63, 992-995 (1989).

\section{Acknowledgements}

The authors thank H. Marx, Th. Weimann and P. Mirovsky for the fabrication of the wafer material and the device. The authors (except V.K.) acknowledge financial support by the German Research Foundation, the Niedersachsen Institutes of Technology and (except T.M., R.J.H., and V.K.) the European Metrology Research Programme (EMRP) within the Joint Research Project 'Quantum Ampere' (JRP SIB07). The EMRP is jointly funded by the EMRP participating countries within EURAMET and the European Union. V.K. has been supported by the Latvian Science Council.

\section{Author contributions}

All authors conceived the research. N.U., T.W. and L.F. carried out the experiments. N.U., F.H. and V.K. analysed the data and wrote the manuscript. V.K. developed the modelling theory and wrote Supplementary Sections A and B. K.P. provided the heterostructure. F.H., H.W.S. and R.J.H. supervised the research. All authors discussed the results and contributed to editing the manuscript.

\section{Additional information}

Supplementary information is available in the online version of the paper. Reprints and permissions information is available online at www.nature.com/reprints. Correspondence and requests for materials should be addressed to N.U.

\section{Competing financial interests}

The authors declare no competing financial interests. 


\section{Partitioning of on-demand electron pairs}

Niels Ubbelohde, Frank Hohls, Vyacheslavs Kashcheyevs, Timo Wagner, Lukas

Fricke, Bernd Kästner, Klaus Pierz, Hans W. Schumacher, and Rolf J. Haug 


\section{A. TUNNELING VERSUS BALLISTIC EMISSION}

We expect that increasing the ramp rate of the electron emission pulse switches the emission from an adiabatic, sequential-tunneling regime to a sudden onset of ballistic propagation, leading to a resolved energy separation between two electrons in a simultaneously emitted pair.

Below we estimate the necessary conditions on characteristic time and energy scales (Sec. A 1) and describe these two distinct electron emission modes using a simple model (Sec. A 2 and A 3). Although not all model parameters can be determined based on available experimental data, the robustness of the calculation outcomes supports our assumptions about the electron source operation regimes that lead to the qualitatively different results of electron pair partitioning described in the paper.

\section{Conditions on the slew rate and tunnel barrier characteristics}

Escape of electrons from the quantum dot in the tunneling regime can be described by emission rate $\Gamma$ which typically depends exponentially on the single-electron energy $E$,

$$
\Gamma(E)=\Gamma_{b} e^{-\left|E-E_{b}\right| / \Delta_{\text {exit }}} .
$$

Here $\Delta_{\text {exit }}$ is an energy scale factor characterizing the exit barrier in the relevant range of emission energies, $\Gamma_{b}$ is the ballistic rate at which confined electrons would escape if the barrier is removed (ie., if $E$ exceeds the barrier height $\left.E_{b}\right) . \Gamma_{b}$ is on the order of the level spacing $\Delta \epsilon$ on the dot, $\Gamma_{b} \sim \Delta \epsilon / \hbar$, and the equation can only be applied in the tunneling regime, $\Gamma \ll \Gamma_{b}$

At sufficiently slow slew rates $\dot{\varepsilon} \equiv d E(t) / d(t)$, the emission from a well-defined state on the quantum dot can be described adiabatically with escape rates (S1) controlled by the time-dependent energy $E(t)$, see Sec. A 2 below. The characteristic time $\tau \equiv \Delta_{\text {exit }} / \dot{\varepsilon}$ for the exponential grow of the tunneling rate must be longer than the inverse ballistic rate $\Gamma_{b}^{-1}$, hence the condition for the adiabatic tunneling emission is

$$
\dot{\varepsilon} \ll \Gamma_{b} \Delta_{\text {exit }}
$$

Taking into account that $\Gamma_{b} \sim \Delta \epsilon / \hbar$, the condition (S2) is the same as the condition for negligible non-adiabtic quantum excitation due to dynamic broadening [V.K. and J.Timoshenko, 
Phys. Rev. Lett. 109, $216801(2012)], \hbar / \tau \ll \Delta \epsilon$.

\section{Sequential tunneling emission (low slew rate)}

As long as the dot remains in the adiabatic tunneling regime, the emission time $t_{e}$ can be estimated [30] by requiring the intergrated out-tunneling rate to be on the order of unity, $\int^{t_{e}} \Gamma(E(t)) d t=\int^{E\left(t_{e}\right)} \dot{\varepsilon}^{-1} \Gamma(E) d E \sim 1$. For a linear ramp, $E(t)=E_{0}+\dot{\varepsilon} t$, and an exponential opening of the tunnel barrier, Eq. (S1), this argument yields an estimate of the emission energy,

$$
E_{\mathrm{em}} \equiv E\left(t_{e}\right)=E_{b}-\Delta_{\text {exit }} \ln \left(\Gamma_{b} \tau\right)
$$

which depends only on the barrier properties and the slew rate (via $\tau$ ), but not on the dc offset $E_{0}$ of the level position. The tunneling approximation for the emission time and energy is consistent under the condition (S2): $\Gamma_{b} \tau \gg 1$ implies $E_{b}-E_{\text {em }} \ll \Delta_{\text {exit }}$ and $\Gamma\left(t_{e}\right) \sim 1 / \tau \ll \Gamma_{b}$.

The energetic width $\Delta E$ of the emitted single-electron packet has contributions [V.K. and J.Timoshenko, Phys. Rev. Lett. 109, 216801 (2012)] both form the stochastic uncertainty $\pm \tau$ of the emission time, $\Delta E \sim \dot{\varepsilon} \tau=\Delta_{\text {exit }}$ and from quantum broadening, $\Delta E \sim \hbar \Gamma\left(t_{e}\right)=$ $\hbar / \tau=\hbar \dot{\varepsilon} / \Delta_{\text {exit }}$, thus we can estimate

$$
\Delta E^{\text {tunnel }} \sim \max \left(\Delta_{\text {exit }}, \hbar \dot{\varepsilon} / \Delta_{\text {exit }}\right)
$$

For the case of two electrons separated by an addition energy gap $\Delta \mu$, similar analysis based on rate equations with time-dependent tunneling rates can be performed. As long as the tunneling rate is determined by the energy of the top-most (first) electron, the emission spectrum will be centered around $E_{\mathrm{em}}$ for both electrons. If $\Delta \mu \gg \Delta_{\text {exit }}$ then the electrons will be well separated in time, with the delay given by the time it takes the tunneling rate of the better-confined (lower energy) electron to reach the value of order $1 / \tau$ after the emission of the loosely confined (higher energy) electron. This time delay can be estimated as $\Delta \mu / \dot{\varepsilon}=\tau \Delta \mu / \Delta_{\text {exit }}$. 


\section{Sudden ballistic emission (high slew rate)}

Choosing a synthesized waveform with a sharp emission pulse enables us to increase the slew rate $\dot{\varepsilon}$ considerably. Sufficiently fast ejection will leave no time for the electrons to tunnel out of the dot before they end up at energies above the potential barrier. In the limit when the adiabatic tunneling condition (S2) is strongly violated, $\dot{\varepsilon} / \Delta_{\text {exit }} \gg \Gamma_{b} \sim \Delta \epsilon / \hbar$, we can use the sudden approximation to describe the transition form a decoupled quantum dot to ballistic propagation.

For a specific model calculation we consider a sudden onset of strong dot-lead coupling in a quantum-impurity-like model of the quantum dot and the edge channel, governed at $t>0$ by the Hamiltonian

$$
\begin{aligned}
\mathcal{H} & =\varepsilon_{c}\left(d_{1}^{\dagger} d_{1}+d_{2}^{\dagger} d_{2}\right)+\left(\Delta \varepsilon+U d_{1}^{\dagger} d_{1}\right) d_{2}^{\dagger} d_{2} \\
& +\sum_{i=1,2} \sqrt{\gamma_{i} /(2 \pi \nu)} \sum_{k}\left(d_{i}^{\dagger} c_{k}+c_{k}^{\dagger} d_{i}\right)+\sum_{k} \epsilon_{k} v_{F} c_{k}^{\dagger} c_{k}
\end{aligned}
$$

Here $\varepsilon_{c}>E_{b}$ is the lowest single-electron energy level right after application of the emission pulse at $t=0 ; \nu$ is the density of states in the lead with a quasi-continuous spectrum $\epsilon_{k}$. $d_{1}, d_{2}$ and $c_{k}$ are the usual fermionic annihilation operators for a single spin species. The level broadenings $\gamma_{1}, \gamma_{2}$ are on the order of $\hbar \Gamma_{b}$ which in turn is expected to be on the order of level-spacing $\Delta \varepsilon$. Pre-emission Coulomb charging energy is described by the Hubbard parameter $U$.

Starting from initial conditions at $t=0$ with two electrons on the dot, $\left\langle d_{1}^{\dagger} d_{1}\right\rangle+\left\langle d_{2}^{\dagger} d_{2}\right\rangle=2$, and a drained Fermi sea, $\left\langle c_{k}^{\dagger} c_{q}\right\rangle=0$, time evolution of the two-particle wavefunction governed by Eq. (S5) can be solved exactly using standard methods. For $t \gg \Gamma_{b}^{-1}$, the solution converges to $|\Psi\rangle=\sum_{k q} \psi_{k q}|k\rangle \otimes|q\rangle$ where $\psi_{k q}=-\psi_{q k}$ and $|k\rangle$ are the single particle states in the lead created by $c_{k}^{\dagger}$. The asymptotic two-particle amplitudes are

$$
\psi_{k q}=\frac{1}{2 \pi \nu \sqrt{2}} \frac{\Delta \varepsilon \sqrt{\gamma_{1} \gamma_{2}}}{\left(\epsilon_{q}-z_{1}\right)\left(\epsilon_{q}-z_{2}\right)\left(\epsilon_{q}+\epsilon_{k}-U-z_{1}-z_{2}\right)}-(q \leftrightarrow k) .
$$

Here $z_{1}$ and $z_{2}$ are the (complex) roots of the secular quadratic equation: $\left(z-z_{1}\right)\left(z-z_{2}\right)=$ $\left(z-\varepsilon_{c}+i \gamma_{1} / 2\right)\left(z-\varepsilon_{c}-\Delta \varepsilon+i \gamma_{2} / 2\right)+\gamma_{1} \gamma_{2} / 4$.

For $\gamma_{1}=\gamma_{2}=\Delta \varepsilon$ (which mimics ballistic propagation over the exit barrier), the joint probability distribution to find one electron at energy $\epsilon_{k}$ and the other at $\epsilon_{q}$ is

$$
\nu^{2}\left|\psi_{k q}\right|^{2}=\frac{\Delta \epsilon^{4}}{8 \pi^{2}} \frac{\left(\epsilon_{k}-\epsilon_{q}\right)^{2}\left[\left(\epsilon_{k}+\epsilon_{q}\right)^{2}+\Delta \varepsilon^{2}\right]}{\left(\epsilon_{k}^{2}+\Delta \varepsilon^{2} / 4\right)\left(\epsilon_{q}^{2}+\Delta \varepsilon^{2} / 4\right)\left[\left(\epsilon_{k}+\epsilon_{q}-U\right)^{2}+\Delta \varepsilon^{2}\right]} .
$$



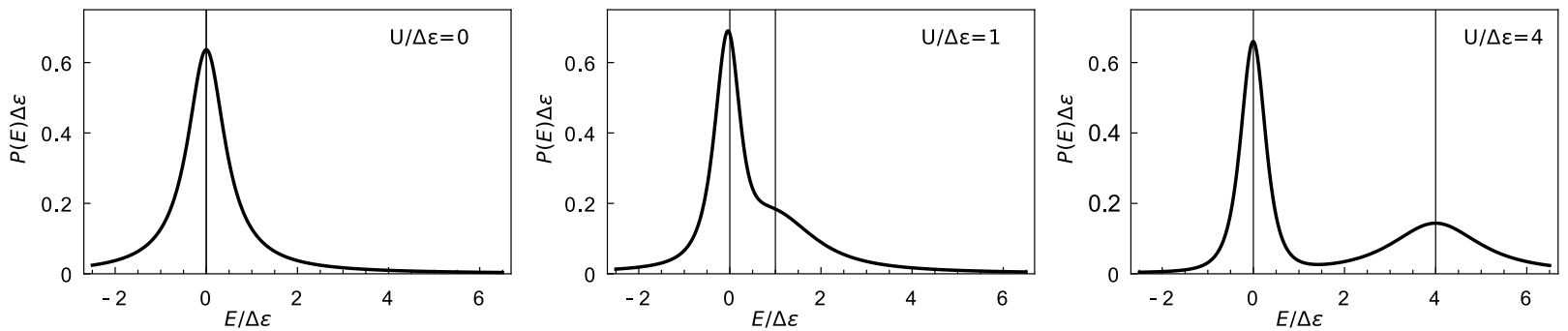

Supplementary Figure 1. The spectrum of single-electron energies $P(E)$ emitted in the sudden approximation for charging energies $U / \Delta \varepsilon=0,1$ and 4 (from left to right). The thin vertical lines mark the positions of $\varepsilon_{c}$ and $\varepsilon_{c}+U$.

This result contains both fermionic $\left(\psi_{k k}=0\right)$ and interactions-induced (for $U>0$ ) correlations. We plot the projected energy spectrum $P\left(\epsilon_{k}\right)=2 \int\left|\psi_{k q}\right|^{2} \nu^{2} d \epsilon_{q}$ for three representative cases in Supplementary Figure 1. The distribution becomes bimodal for sufficiently large interaction energy, meaning that electrons can be separated in energy. The widths of the peaks are on the order of quantum broadening which we equate with the level spacing on the dot:

$$
\Delta E^{\text {sudden }} \sim \Delta \varepsilon
$$

Note that our energy width estimates on the tunneling (slow) and the sudden (fast) sides of the transition driven by the slew rate $\dot{\varepsilon}$ (see the condition $(\mathrm{S} 2)$ ) agree if extrapolated into the crossover region, $\Delta E^{\text {tunnel }} \sim \Delta E^{\text {sudden }}$ if $\hbar \dot{\varepsilon} \sim \Delta_{\text {exit }} \Delta \varepsilon$.

The simple two-orbital model analyzed here does not include higher excited states on the dot, hence it can not predict the line-shapes quantitatively (e.g., the higher width of the second peak at $\varepsilon_{c}+U$ in Supplementary Figure 1 depends on the exact assumptions about $\gamma_{1}, \gamma_{2}$ and $\left.\Delta \varepsilon\right)$. Nevertheless, the calculation makes clear that interactions in the source may induce a bimodal energy distribution of the emitted electrons in case of a sharp ejection pulse as employed in the experiment.

\section{B. COUNTING STATISTICS OF ELECTRON PAIR PARTITIONING UNDER LINEAR SCATTERING}

Here we assess the effect of initial correlations on the counting statistics of two electrons partitioned by an energy barrier which does not induce additional two-body correlations. 
Specifically, we test the following combination of model assumptions: (a) absence of twobody interactions at the barrier; (b) single chiral orbital channel per spin projection $\sigma=\uparrow, \downarrow$, and (c) monotonic dependence of the single-electron scattering probability $T_{\sigma}(E)$ on the energy $E$ as tuned by the barrier-creating gate.

Assumption (a) implies that the quantum numbers that diagonalize the single-particle scattering matrix remain good for the two-particle transmission. This allows one to express the two-particle scattering probabilities as

$$
\begin{aligned}
& p_{2}=\sum_{k q} \sum_{\sigma \sigma^{\prime}} \rho_{k \sigma q \sigma^{\prime}} T_{k \sigma} T_{q \sigma^{\prime}}, \\
& p_{0}=\sum_{k q} \sum_{\sigma \sigma^{\prime}} \rho_{k \sigma q \sigma^{\prime}}\left(1-T_{k \sigma}\right)\left(1-T_{q \sigma^{\prime}}\right),
\end{aligned}
$$

where $0 \leq T_{k \sigma} \leq 1$ are the transmission coefficients of the scattering eigenmodes and $\rho_{k \sigma q \sigma^{\prime}}$ is the diagonal element of the two-particle density matrix of the incoming state. The third component of the statistics is $p_{1}=1-p_{0}-p_{2}$. The necessary conditions on $\rho_{k \sigma q \sigma^{\prime}}$ are

non-negativity, normalization $\left(\sum_{k \sigma q \sigma^{\prime}} \rho_{k \sigma q \sigma^{\prime}}=2\right)$, and exchange symmetry $\left(\rho_{k \sigma q \sigma^{\prime}}=\rho_{q \sigma^{\prime} k \sigma}\right)$. (The latter is due to electrons being identical particles, not necessarily in identical states).

\section{Examples of enhanced partitioning due to either Fermi correlations or an energy} gap

Here we show that the linear scattering theory expressed by assumptions (a)-(c) and Eq. (S9) does allow enhanced partitioning $\left(p_{1}>50 \%\right)$ both for statistics-dominated or interactions-induced initial correlations.

Using Eq. (S9) for the partitioning statistics, and the diagonal elements of the density matrix (S7) from a model calculation of Sec. A 3 with $T\left(\epsilon_{k}\right)$ being a sharp $\left(\Delta_{b} \ll \Delta \epsilon, U\right)$ function of energy gives the results shown in Supplementary Figure 2.

We can see that for $\Delta \varepsilon \gtrsim \Delta_{b}$ electrons can be efficiently partitioned by either pure statistics $(U \ll \Delta \varepsilon)$ or on-the-dot interacitons $(U \gg \Delta \varepsilon)$. Energy separation is not a neccessary condition for enhanced partitioning, as can be seen from the $U=0$ case in Supplementary Figures 1 and 2 . 

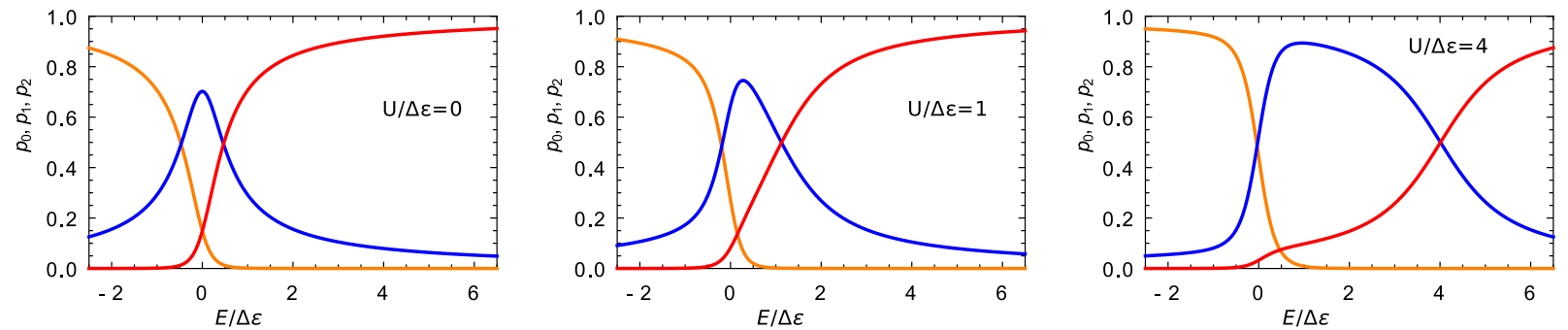

Supplementary Figure 2. Counting statistics for the pair partitioning, calculated in the sudden emission approximation for the same model parameters as in Supplementary Figure 1. Orange, blue, and red curves depict $p_{0}, p_{1}$ and $p_{2}$, respectively, as functions of the sharp cut-off threshold $E$ of the detector barrier.

\section{Poisson binomial statistics for minimally entangled electrons}

Here we consider only minimally entangled states for a propagating electron pair and show that under the assumptions (a)-(c) of interactions-free scattering (listed at the beginning of Supplementary Information B) such states cannot violate the Poisson binomial constraint on the partitioning statistics, $\sqrt{p_{0}}+\sqrt{p_{2}} \leq 1$. The arguments here are more general than a specific model discussed in Secs. A 3 and B 1.

For a pure state composed of two orthogonal spinorbitals, $|\Psi\rangle=\frac{1}{\sqrt{2}}(|a\rangle \otimes|b\rangle-|b\rangle \otimes|a\rangle)$ (a Slater determinant), one has $\rho_{k \sigma q \sigma^{\prime}}=\mid\left\langle\Psi\left|\left(|k \sigma\rangle \otimes\left|q \sigma^{\prime}\right\rangle\right)\right|^{2}=\frac{1}{2}\left|\langle a \mid k \sigma\rangle\left\langle b \mid q \sigma^{\prime}\right\rangle-\langle b \mid k \sigma\rangle\left\langle a \mid q \sigma^{\prime}\right\rangle\right|^{2}\right.$ and the counting statistics (S9) become

$$
p_{2}=\operatorname{det}\left[\begin{array}{cc}
\langle a|\hat{T}| a\rangle & \langle a|\hat{T}| b\rangle \\
\langle b|\hat{T}| a\rangle & \langle b|\hat{T}| b\rangle
\end{array}\right], \quad p_{0}=\operatorname{det}\left[\begin{array}{cc}
1-\langle a|\hat{T}| a\rangle & \langle a|\hat{T}| b\rangle \\
\langle b|\hat{T}| a\rangle & 1-\langle b|\hat{T}| b\rangle
\end{array}\right],
$$

where $\langle x|\hat{T}| y\rangle$ are matrix elements of a single-particle operator $\hat{T}=\sum_{k \sigma}|k \sigma\rangle T_{k \sigma}\langle k \sigma|$. Expressing the determinants in terms of products of eigenvalues, we arrive at Poisson binomial distribution $p_{2}=T_{a} T_{b}$ and $p_{0}=\left(1-T_{a}\right)\left(1-T_{b}\right)$. It easily follows from $0 \leq p_{i} \leq 1$ that the eigenvalues satisfy $0 \leq T_{a}, T_{b} \leq 1$. An equivalent derivation utilizing a generating function for the counting statistics can be found in [24, 25].

The model discussed in Sec. B 1 provides an example of a minimally entangled state for $U=0$ : equation (S6) is reproduced by taking $\langle k \mid a\rangle=(\gamma / \pi)^{1 / 2} /\left(\epsilon_{k}+i \gamma\right)$ and $\langle k \mid b\rangle=$ $(\gamma / \pi)^{1 / 2}\left(\gamma+i \epsilon_{k}\right) /\left(\gamma-i \epsilon_{k}\right)^{2}$ with $\gamma \equiv \Delta \varepsilon / 2$. The deviations from the simple binomial statistics seen in the first panel of Supplementary Figure 2 in this language are explained 
by the overlap of the initially orthogonal wavefunctions $|a\rangle$ and $|b\rangle$ after the transmission, $\langle b|\hat{T}| a\rangle=\pi^{-1} \gamma /(\gamma+i E)$.

Eigenstates of the total spin operator that carry minimal entanglement are the spin singlet state with a doubly occupied orbital and the fully polarized two-orbital triplet states,

$$
\begin{aligned}
|S\rangle & \propto(|\uparrow \downarrow\rangle-|\downarrow \uparrow\rangle)|\psi \psi\rangle, \\
\left|T_{\sigma}\right\rangle & \propto|\sigma \sigma\rangle\left(\left|\psi_{1} \psi_{2}\right\rangle-\left|\psi_{2} \psi_{1}\right\rangle\right) .
\end{aligned}
$$

Since $|S\rangle$ and $\left|T_{\sigma}\right\rangle$ are expressible by a single Slater determinant, their partitioning by an interactions-free barrier will also result in a generalized binomial distribution, regardless of the barrier-induced overlap $\langle a|\hat{T}| b\rangle$. If the scattering is spin-independent, then the same applies to the non-spin-polarized component of a two-orbital triplet, $\left|T_{0}\right\rangle \propto$ $(|\uparrow \downarrow\rangle+|\downarrow \uparrow\rangle)\left(\left|\psi_{1} \psi_{2}\right\rangle-\left|\psi_{2} \psi_{1}\right\rangle\right)$.

Hence, only the states that carry more correlations than required by the entanglement due to exchange statistics may violate the constraint $\sqrt{p_{0}}+\sqrt{p_{2}} \leq 1$ under the singleparticle scattering assumption (a), thus ruling out minimal-entanglement interactions-free explanations for the correlation (bunching) anomaly reported in the paper.

\section{Monotonicity argument}

Here we state implications of the assumptions (a)-(c) defined in the beginning of Sec. B for the partitioning statistics (S9) of an arbitrarily correlated initial density matrix.

Assumption (b) establishes that the orbital indices $k$ are not degenerate in energy. If $T_{k \sigma}=T_{\sigma}\left(\epsilon_{k \sigma}+E\right)$ where $\epsilon_{k \sigma}$ is the kinetic energy of the plane-wave mode with respect to a fixed reference level, and $E$ is tuned monotonically by the detector barrier gate, then by assumption (c) and by the nonnegativity of $\rho$, the probabilities $p_{2}(E)$ and $p_{0}(E)$ have to be monotonic in $E$ as well.

Therefore, models considering electron-electron interactions in the source only (like the example in Sec. A 3) may not be sufficient to explain the entirety of observation reported in the paper. 


\section{SUPPLEMENTARY INFORMATION}
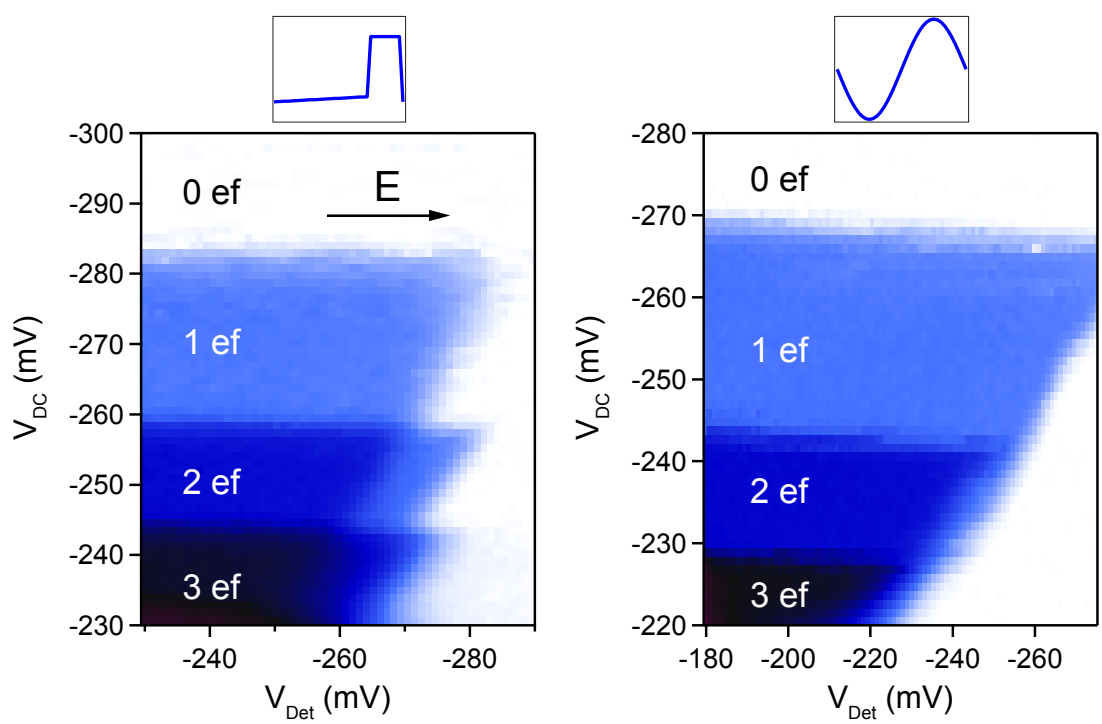

Supplementary Figure 3. Transmitted current $\mathrm{I}_{\mathrm{T}}$ as a function of the exit barrier $\left(\mathrm{V}_{\mathrm{DC}}\right)$ and the detector barrier $\left(\mathrm{V}_{\text {Det }}\right)$ with the entrance gate driven by a pulse-waveform (left) and a sine-waveform (right).

\section{DEPENDENCE OF THE ENERGY DETECTION ON THE WAVEFORM}

To illustrate the effect of a sharp ejection pulse we compare intensity plots of the transmitted current as a function of the exit gate and the detector gate (Supplementary Figure 3). By tuning the exit gate a larger number of electrons are sourced per waveform cycle and the emission energy follows the exit barrier height. The detector gate dependence reflects the energy distribution of the emitted electrons. Switching the waveform from a sine to an optimized waveform with a sharp ejection pulse leaves the behavior in the regime of 1ef unchanged. However, in the 2ef regime a stepwise extension towards higher energies is observed, c.f. Fig. 3c. For a sine waveform no such step is visible. 